\title{
FEBRE PURPÚRICA BRASILEIRA. CARACTERIZAÇÃo RÁPIDA DAS CEPAS INVASORAS DE HAEMOPHILUS AEGYPTIUS
}

\author{
M.C.C. BRANDILEOVE (1); V.S.D. VIEIRA (1); M.L.C. TONDELLA (1); C.T. SACCHI (1); 1.M. LANDCRAF (1);
} R.C. ZANELLA (1); W.F. BIBB (2); K. IRINO (1) \& GRUPO DE ESTUDO DA FEBRE PURPÚRICA BRASILEIRA

\section{RESUMO}

Cepas de H. aegyptius isoladas em surtos de Febre Purpúrica Brasileira (FPB) no Brasil, foram caracterizadas pelo método de aglutinação em lâmina utilizando um anti-soro produzido com cepa de $\mathbf{H}$. aegyptius isolada de cultura de sangue de paciente com FPB. Através desse método foi possível identificar cepas de H. aegyptius responsáveis por surtos de conjuntivite com características antigênicas iguais às cepas isoladas de FPB. A sensibilidade e especificidade da soroaglutinação em lâmina foi de $97,7 \%$ e $89,6 \%$ respectivamente, podendo ser utilizado como método de triagem em estudos de conjuntivites purulentas, para detectar cepas invasivas de $\mathbf{H}$. aegyptius associadas a FPB, possibilitando assim a implantação de medidas que ampliem a eficiência na prevenção e na vigilância epidemiológica da doença.

UNITERMOS: Haemophilus aegyptius: Febre purpúrica brasileira, Diagnóstico laboratorial; Soroaglutinação em lâmina.

\section{INTRODUÇÃO}

A Febre Purpúrica Brasileira (FPB) é uma doença sistêmica com caráter geralmente epidêmico, apresentando-se associada a surtos de conjuntivite purulenta. Acomete crianças na faixa etária de 2 meses a 10 anos, caracterizando-se clinicamente por febre alta, vômitos e dor abdominal, evoluindo para o aparecimento de púrpura, colapso vascular e necrose da pele ${ }^{3,4,5}$.

No período de 1986 a 1988, cepas de Haemophilus aegyptius foram isoladas de sangue ou de líquido cefalorraquidiano de 17 pacientes com FPB residentes em regiões de Ribeirão Preto, Presidente Prudente e São José do Rio Preto, no Estado de São Paulo, Brasil6,11. Estes, juntamente com as recentes descrições em Israel ${ }^{21}$ e Austráliai ${ }^{17}$ de casos de endocaraite e FPB, respectivamente, são os únicos relatos de doença sistêmica causada por esse microrganismo.

Epidemias de conjuntivite purulenta que têm como agente etiológico o $H$. aegyptius ocorrem frequentemente em paises de clima quente1.No Brasil, casos de FPB associados a surtos de conjuntivite por esta bactéria tem sido observados desde 1984 na região Oeste do Estado de São Paulo4,5,9 e em 1988 no município da Capital.

$O$ H. influenzae e o H. aegyptius foram considerados por alguns autores como duas espécies distintas, devido a diferenças em suas características morfológicas, bioquímicas, exigências nutricionais, capacidade de aglutinar hemácias " $O$ " e variação na sua vi-

(1) Seção de Bacteriologia do Insiitulo Adolfo I.uk, São Paulo, Brasil.

(2) Centers for Disease Control, Atlanta, Georgia, USA.

Endereço para correspondência: Dra. Maria Cristina de Cunto Brandileone. Insituto Adolfo Lulz, Seçào dẹ Bacteriolog̣ia. Ar. Dr. Arnaldo, 355. (EP 01246, Sào Paulo, SP, Brasil. 
BRANDILEONE, M.C.C.; VIEIRA, V.S.D.; TONDELLA, M.L.C.; SACCHI, C.T.; LANDGRAF, I.M.; ZANELLA, R.C.; BIBB, W.F.; IRINO, K. \& GRUPO DE ESTUDO DA FEBRE PURPÚRICA BRASILEIRA - Febre purpúrica brasileira. Caracterização rápida das cepas invasoras de Haemophilus aegyptius. Rev. Inst. Med. trop. S. Paulo, 31(4):221-227, 1989.

rulência8,13,16. Outros autores não consideraram estas características suficientes para diferenciar as duas espécies, classificando o H. aegyptius como biotipo III do $\mathbf{H}$. influenzae. Estudos genéticos de hibridização DNA/DNA20 e de transformação sugerem que o H. aegyptius e o $H$. influenzae constituem uma única espécie 2,14,15. Atualmente, está sendo proposta a designação de H. influenzae biogrupo aegyptius para o $\mathbf{H}$. aegyptius?

Estudos envolvendo a caracterização do perfil enzimático, das proteinas totais, dos padróes de restrição do gene rDNA e dos plasmídios sugerem que as cepas de $\mathbf{H}$. aegyptius invasivas apresentam características específicas que as diferenciam de outras cepas ${ }^{7}$.

O estudo de marcadores epidemiológicos

do H. aegyptius é de fundamental importância para a vigilância epidemiológica da FPB, no sentido de se identificar os surtos de conjuntivite purulenta causadas por cepas invasivas de $H$. aegyptius.

Este trabalho tem por objetivo caracterizar cepas invasivas de $\mathbf{H}$. aegyptius, pelo estudo de seus antígenos de superfície pela técnica de soroaglutinação em lâmina, utilizando antisoro produzido com uma cepa de $\boldsymbol{H}$. aegyptius isolada de paciente com FPB.

\section{MATERIAL E MÉTODOS}

Foram estudadas 331 cepas de Haemophilus sp distribuidas em $\mathbf{6}$ grupos discriminados abaixo:

Grupo 1 - 22 cepas de $H$. aegyptius, isoladas de 17 casos confiimados de FPB*, em diferenles municípios do Estado de São Paulo (Promissão em 1984, Serrana, Guatapará, Fartura, São José do Rio Preto e Presidente Prudente em 1986, Serra Azul em 1988).

Gïupo 2 - 26 cepas de H. aegyptius (25 cepas isoladas de secreçoes conjuntivais e 1 de orofaringe) de pacientes que apresentavam exclusivamente conjuntivite, procedentes de municipios do Estado de São Paulo onde ocorreran na mesma época casos confirmados de FPB (Pro- missão em 1984 e 1985, São José do Rio Preto e Presidente Prudente em 1986, Serrana em 1986 e 1988, Serra Azul em 1988).

Grupo $3-20$ cepas de $\mathbf{H}$. aegyptius ( 20 cepas isoladas de secreções conjuntivais) isoladas de pacientes que apresentavam exclusivamente conjuntivite, procedentes de municípios do Estado de São Paulo onde ocorreram casos suspeitos de FPB** (Garça, Bauru e Lins em 1985 e Piracicaba em 1986, Pradópolis em 1987, São José do Rio Preto e Nova Granada em 1988).

Grupo $4-53$ cepas de $\mathbf{H}$. aegyptius isoladas de pacientes que apresentavam exclusivamente conjuntivite (51 cepas isoladas de secreções conjuntivais e 2 de orofaringe) procedentes de municipios do Estado de São Paulo onde não ocorreram casos confirmados ou casos suspeitos de FPB (Bastos em 1985, Restinga, Barretos, Vista Alegre do Alto, Dois Córregos, Ribeirão Preto, São Paulo e Guariba em 1986, Promissão e Guariba em 1987, Tanabi, Promissão, Fernandópolis e Ribeirão Preto em 1988).

Grupo $5-8$ cepas de $H$. aegyptius procedentes de outros países, e 2 cepas padrões.

- H. aegyptius de outros países:

$\mathrm{H}-32974$ - isolada de sangue de um caso de FPB ocorrido na Austrália.

F-3330, 172a, 175a, 145a, 180a, KC-1018 isoladas nos Estados Unidos da secreção da conjuntiva.

F-3331, isolada no Egito, da secreção da conjuntiva.

-- Cepas padrões: H. aegyptius NCTC - 8135

H. influenzae $\mathrm{GB}-3291$

Grupo 6 - 200 cepas de Haemophilus sp, 163 cepas de H. influenzae ( 80 de secreção conjuntival e 83 de orofaringe) e 37 cepas de H. parainfluenzae ( 6 de secreção conjuntival, 31 de secreçào de orofaringe), isoladas em 1987 e 1988 durante surtos de conjuntivite, procedentes de diferentes municípios do Estado de São Paulo, onde ocorreram casos confirmados e casos suspeitos de FPB e onde não ocorreram casos.

Todas as cepas foram mantidas a $-70^{\circ} \mathrm{C}$, cm caldo Levinthal acrescidos de $15 \%$ de glicerol cu mantidas liofilizadas a $+4^{\circ} \mathrm{C}$.

Confirmados elinicamente e pela presença dc $\mathbf{H}$ aegyptius no sangue ou líquido cefalorraquidiano ou na lesão purpúrica da pele. ** Clinicamente confirmado, sem confirmaçào laborato-
rial. 
BRANDILEONE, M.C.C.; VIEIRA, V.S.D.; TONDELLA, M.L.C.; SACCHI, C.T.; LANDGRAF, I.M.; ZANELLA, R.C.; BIBB, W.F.; IRINO, K. \& GRUPO DE ESTUDO DA FEBRE PURPÚRICA BRASILEIRA - Febre purpúrica brasileira. Caracterização rápida das cepas invasoras de Haemophilus aegyptius. Rev. Inst. Med. trop. S. Paulo, 31(4):221-227, 1989.

Foram produzidos 1 anti-soro com a cepa de H. aegyptius e 6 anti-soros contra os antígenos capsulares dos sorotipes de $\mathbf{H}$. influenzae.

$\mathrm{O}$ soro anti-H. aegyptius foi produzido com a cepa 254/86 amostra esta, isolada do sangue de um paciente de FPB. Este soro foi absorvido e utilizado da seguinte forma:

a) S-254 - soro anti-H. aegyptius produzido com a cepa $254 / 86$, isolada a partir de cultura de sangue de um caso de FPB.

b) S-254/254F - soro anti-H. aegyptius produzido com a cepa $254 / 86$ e absorvido com a mesma cepa fervida a $100^{\circ} \mathrm{C}$ em banhomaria por 2 horas.

c) $S-254 / 329$ - soro anti-H. aegyptius produzido com a cepa $254 / 86$ e absorvido com a cepa $329 / 86$, também isolada de liquido cefalorraquidiano de um caso de FPB.

d) Soros capsulares a, b, c, d, e, f, produzidos com as cepas padrões dos tipos capsulares de H. influenzae SM-2, GB-3291, SM-72, SM6 , SM-7, SM-8, respectivamente.

O anti-soro de $\mathbf{H}$. aegyptius foi produzido em coelhos albinos adultos com peso igual ou superior a $3 \mathrm{~kg}$. A partir de uma suspensão bacteriana contendo aproximadamente 1,2 $\times 10^{4}$ bactérias/ml (escala 4 de MacFarland), e morta pela formalina $0,5 \%$ da seguinte forma:

a) suspensão bacteriana de $6 \times 10^{8}$ bactérias $/ \mathrm{ml}$ (escala 2 de MacFarland) com adjuvante completo de Freund; $1 \mathrm{ml}$ deste antígeno foi inoculado por via intradérmica no dorso do animal, em 15 diferentes pontos.

b) 30 dias após a inoculação intradérmica, 0,5 $\mathrm{ml}$ da mesma suspensão bacteriana com adjuvante incompleto de Freund foi inoculado em uma única dose por via intramuscular na coxa posterior.

c) 7 dias após, 1 dose de $1 \mathrm{ml}$ da suspensão bacteriana de $9 \times 10^{\times}$bactérias/ml (escala 3 de MacFarland) sem adjuvante, foi inoculada semanalmente, durante 4 semanas por via endovenosa.

Uma semana após a última inoculação, os animais foram sangrados e os anti-soros separados dos coágulos, foram conservados a $-20^{\circ} \mathrm{C}$, adicionado de $0,05 \%$ de azída sódica.

Os anti-soros capsulares de $\mathbf{H}$. influenzae foram obtidos segundo a metodologia descrita no Procedural Manual for Production of Bacterial, Fungal, and Parasitic Reagent ${ }^{10 .}$.
As absorções do anti-soro S-254 foram feitas com a mistura de 1 volume de anti-soro diluído $1: 2$, com igual volume de células bacterianas cultivadas em caldo Levinthal por 18 horas, sob agitação em estufa a $36^{\circ} \mathrm{C}$, coletadas por centrifugação e lavadas, uma vez, em PBS, $0,1 \mathrm{M}, \mathrm{pH} 7,2$. A mistura anti-soro-bactérias foi homogeneizada e deixada por 2 horas a $37^{\circ} \mathrm{C}$ e a seguir 18 horas a $4^{\circ} \mathrm{C}$. Os anti-soros absorvidos foram obtidos após centrifugação a 6.000rpm por 20 minutos a $+4^{\circ} \mathrm{C}$.

As titulações foram feitas pela técnica de soroaglutinação em lâmina, utilizando como antigeno, uma densa suspensão bacteriana contendo aproximadamente $12 \times 10^{y}$ bactérias $/ \mathrm{ml}$, e adicionada de formalina a $0,5 \%$. Foi utilizada soluçâo salina a $2 \%$ para o controle da rugosidade das cepas bacterianas. As cepas de $\mathbf{H}$. aegyptius foram testadas em diluiçōes seriadas de 1:2 a 1:1024 dos soros S-254, S-254/254F e S-254/329, tendo sido consideradas como diluiçôes de uso aquelas que apresentaram uma forte aglutinação $(+++$ ou ++++$)$. Os 6 antisoros capsulares de $\mathbf{H}$. influenzae nas suas respectivas diluições de uso, foram também incluídos neste estudo.

O teste de aglutinação foi comparado com os padrōes de restrição do gene rDNA que caracterizam as cepas de $\mathbf{H}$. aegyptius associados a casos de FPB, pertencentes aos padrões 3 e 412.

\section{RESULTADOS}

Os resultados das titulações dos anti-soros S-254, S-254/254F e 254/329 com a cepa n. 254/86 estão na tabela 1 .

O título do anti-soro S-254 foi de 1:1024 e do anti-soro S-254/254F foi de 1:256; com o anti-soro S-254/329 não houve aglutinação em nenhuma diluição com a cepa $254 / 86$.

Os resultados das soroaglutinações das 22 cepas de $\mathbf{H}$. aegyptius do grupo 1 estão apresentados na tabela 2. Nesta tabela observamos que todas as cepas de H. aegyptius isoladas de casos de FPB no Brasil aglutinaram com o anti-soro S-254, em tílulos que variaram de 1:64 a 1:256 $(++++)$ e com o anti-soro S-254/254F, entre 1:32 e $1: 128(++++)$; nenhuma cepa do grupo 1 aglutinou com o anti-soro S-254/329.

Pela tabela 3 onde estão relacionados o número e percentual de cepas de $\mathbf{H}$. aegyptius, pertencentes aos grupos 1, 2, 3 e 4, verificamos 
BRANDILEONE, M.C.C.; VIEIRA, V.S.D.; TONDELL.A, M.L.C.; SACCHI, C.T.; LANDGRAF, I.M.; ZANELLA, R.C.; BIBB, W.F.; IRINO, K. \& GRUPO DE ESTUDO DA FEBRE PURPURICA BRASILEIRA — Febre purpúrica brasileira. Caracterizaçào rápida das cepas invasoras de Haemophilus aegyptius. Rev. Inst. Med. trop. S. Paulo, 31(4):221-227, 1989.

\section{TABELA 1}

Títulos homólogos aglutinantes dos anti-soros S-254, S-254/254F e S-254/329 obtidos com a cepa n? 254/86 de H. aegyptius

\begin{tabular}{|c|c|c|c|c|c|c|c|c|c|c|}
\hline \multirow{2}{*}{ Soros } & \multicolumn{10}{|c|}{ Diluições } \\
\hline & $1: 2$ & $1: 4$ & $1: 8$ & $1: 16$ & $1: 32$ & $1: 64$ & $1: 128$ & $1: 256$ & $1: 512$ & $1: 1024$ \\
\hline$S-254(1)$ & ++++ & ++++ & ++++ & ++++ & ++++ & ++++ & +++ & +++ & ++ & + \\
\hline $\mathrm{S}-254 / 254 \mathrm{~F}(2)$ & ++++ & ++++ & ++++ & ++++ & ++++ & +++ & +++ & + & - & - \\
\hline$S-254 / 329(3)$ & - & - & - & - & - & - & - & - & - & - \\
\hline $\begin{array}{l}(-) \\
(+) \\
(++) \\
(+++) \\
(++++)\end{array}$ & \multicolumn{10}{|c|}{$\begin{array}{l}\text { Ausência de aglutinação } \\
\text { Aglutinação incompleta, muito fraca } \\
\text { Aglutinação incompleta, fraca } \\
\text { Aglutinação completa, forte } \\
\text { Aglutinação completa, muito forte }\end{array}$} \\
\hline $\begin{array}{l}\text { (1) S- } 254 \\
\text { (2) S- } 254 / 254 \mathrm{~F} \\
\text { (3) } \mathrm{S}-254 / 329\end{array}$ & \multicolumn{10}{|c|}{$\begin{array}{l}\text { soro preparado com a cepa no } 254 \text { - H. aegyptius isolada de sangue de um caso de FPB. } \\
\text { soro S-254 absorvido com a amostra homóloga fervida de H. aegyptius no } 254 \text {. } \\
\text { soro S- } 254 \text { absorvido com antígeno não fervido da cepa no } 329 / 86 \text { - H. aegyptius isolada de um caso } \\
\text { de FPB. }\end{array}$} \\
\hline
\end{tabular}

TABELA 2

Aglutinação de cepas de H. aegyptius do grupo 1 isoladas de 17 casos de FPB, segundo os anti-soros e a origem das cepas

\begin{tabular}{lcccc}
$\begin{array}{l}\text { Material } \\
\text { biológico }\end{array}$ & $\begin{array}{c}\text { Número } \\
\text { Cepas }\end{array}$ & $\begin{array}{c}\mathrm{S}-254 \\
(1 / 64)\end{array}$ & $\begin{array}{c}\mathrm{S}-254 / 254 \mathrm{~F} \\
(1 / 32)\end{array}$ & $\begin{array}{c}\mathrm{S}-254 / 329 \\
\text { (negativo) }\end{array}$ \\
\hline Sangue & 13 & $13 / 13$ & $13 / 13$ & $13 / 13$ \\
LCR & 4 & $4 / 4$ & $4 / 4$ & $4 / 4$ \\
Secreção da conjuntiva & 2 & $2 / 2$ & $2 / 2$ & $2 / 2$ \\
Secreção da orofaringe & 2 & $2 / 2$ & $2 / 2$ & $2 / 2$ \\
Raspado de pele & 1 & $1 / 1$ & $1 / 1$ & $22 / 22$ \\
\hline Total de cepas & 22 & $22 / 22$ & $22 / 22$ & $1 / 1$ \\
\hline
\end{tabular}

TABELA 3

Número e percentual de cepas de $\mathbf{H}$. aegyptius dos grupos 1,2,3 e 4 segundo os resultados das aglutinações com as antisoros $\mathrm{S}-254$ e padrões de restrição do gene rDNA.

\begin{tabular}{cccccc}
\hline $\begin{array}{c}\text { Grupo das } \\
\text { cepas }\end{array}$ & $\begin{array}{c}\text { Número de } \\
\text { cepas estudadas }\end{array}$ & $\begin{array}{c}\text { Aglutinação } \\
\text { rDNA +* }\end{array}$ & $\begin{array}{c}\text { Aglutinação } \\
\text { rDNA +* }\end{array}$ & $\begin{array}{c}\text { Aglutinação + } \\
\text { rDNA - ** }\end{array}$ & $\begin{array}{c}\text { Aglutinação - } \\
\text { rDNA - ** }\end{array}$ \\
\hline 1 & 22 & $22(100 \%)$ & $0(0,0 \%)$ & $0(0,0 \%)$ & $0(0,0 \%)$ \\
2 & 26 & $16(61,5 \%)$ & $0(0,0 \%)$ & $2(7,7 \%)$ & $8(30,8 \%)$ \\
3 & 20 & $3(15,0 \%)$ & $1(5,0 \%)$ & $2(10,0 \%)$ & $14(70,0 \%)$ \\
4 & 53 & $2(3,8 \%)$ & $0(0,0 \%)$ & $4(7,5 \%)$ & $47(88,7 \%)$ \\
\hline
\end{tabular}

(1) - cepas isoladas de casos confirmados de FPB

(2) - cepas isoladas em municípios com casos confirmados de FPB

(3) - cepas isoladas em municípios com casos suspeitos de FPB

(4) - cepas isoladas em municípios sem casos de FPB

* IDNA + perfil de restrição dos tipos 3 ou 4 característicos das cepas invasivas

** rDNA - perfil de restrição diferentes dos tipos 3 ou 4 
BRANDILEONE, M.C.C.; VIEIRA, V.S.D.; TONDELLA, M.L.C.; SACCHI, C.T.; LANDGRAF, I.M.; ZANELLA, R.C.; BIBB, W.F.; IRINO, K. \& GRUPO DE ESTUDO DA FEBRE PURPÚRICA BRASILEIRA - Febre purpurica brasileira. Caracterização rápida das cepas invasoras de Haemophilus aegyptius. Rev. Inst. Med. trop. S. Paulo, 31(4):221-227, 1989.

que os resultados das soroaglutinaçoes e dos perfis de restriçào foram concordantes en $92,5 \%$ das cepas esludadas, e que somente 8 cepas soroaglutinantes possuiam perfis de restricão diferentes dos tipos 3 ou 4 assim como 1 cepat linha perfil de rDNA invasor e foi não aglulinante. Verificamos também que nos municipios onde ocorreram casos confirmados de FPB $61,5 \%$ das cepas isoladas da secreçâ da conjuntiva e ou orofaringe de pacientes que apresentavam exclusivamente conjuntivites, apresentaram aglutinação positiva e padrão de reslrição caracterísı ico de cepas invasivas, enquan10 que apenas $3,8 \%$ de cepas isoladas nos municipios onde não ocorreram casos de FPB apresentam o mesmo perfil. Por outro lado $88,7 \%$ das cepas isoladas nos municipios onde não correram casos de FPB, não aglutinaram com o soro S-254 e apresentaram perfil de restrição do gene rDNA diferentes dos tipos 3 e 4.

As cepas de H. aegyptius do grupo 5, onde inclusive estava a cepa isolada de un caso de FPB ocorrido na Austrália, não aglutinaram com os anti-soros S-254 e S-254/254F.

Nenhuma cepa de Haemophilus sp pertecente ao grupo 6 aglutinou com o anti-soro S254 na diluição de uso $(1: 64)$.

Todas as cepas de $\mathbf{H}$. aegyptius pertecentes aos grupos $1,2,3,4$ e 5 foram testados conıra lodos os anti-soros capsulares de $\mathbf{H}$. influenzae resultando em aglutinação negativa.

A sensibilidade do método de soroaglutinação em lâmina foi de $97,7 \%$ e a especificidade de $89,6 \%$.

\section{DISCUSSÃO}

O padrão antigênico da espécie tipo do gênero H. influenzae é bem conhecidols devido seu grande interesse na clínica de doenças infecciosas, definindo-se pela presença de uma capsula polissacarídica que determina os sorotipos a, b, c, d, e, f. Com relação ao H. aegyptius poucos esiudos foram realizados sobre a sua estrutura antigênica e consequentemente não se tem um esquema sorológico estabelecido.

PITTMAN \& DAVIS', em 1950, estudaram os determinantes antigênicos de H. aegyptius isolados durante um surto de conjuntivite no Texas, e mostraram que cepas de H. aegyptius são antigenicamente diferentes de cepas de
H. influenzae, formando um grupo sorológico relativamente homogêneo. As reações cruzadas sugeriam a presença de muitos componentes antigênicos, distribuidos de modo desigual entre as diferentes cepas de $\mathbf{H}$. aegyptius. Cepas de H. aegyptius que apresentavam o mesmo perfil aglutinante com diferentes anti-soros foram classificados em grupos epidemiologicamente relacionados.

Em nosso trabalho, o título elevado do anli-soro S-254 de H. aegyptius e a absorção do mesmo com a amostra homóloga fervida suge+ rem a presença de determinantes antigênicos de natureza termo lábil na superficie bacteriana. $\mathrm{O}$ soro S-254/329 (Tabela 1) mostrou que as 2 cepas são muito semelhantes, uma vez que a cepa $329 / 86$ foi capaz de esgotar o soro S-254.

As cepas de H. aegyptius isoladas de casos de FPB ocorridos no Estado de São Paulo, Brasil, se caracterizaram por apresentarem o mesmo perfil de aglutinação com os três anti-soros, sugerindo uma constituição antigênica semelhante, enquanto que cepa padrão de H. aegyptius e aquelas originárias de outros paises, inclusive a cepa isolada de um caso de FPB ocorrido na Austrália, não apresentaram os componentes antigênicos específicos das cepas isoladas no Brasil. Uma cepa con características específicas parece ter sido o agente etiológico da FPB no Brasil, desde 1984.

O H. aegyptius sempre foi considerado responsável somente por surtos epidêmicos de conjuntivite purulenta'; a modificação das cepas de H. aegyptius associados a FPB poderia estar relacionada entre outros fatores, à aquisição destes novos componentes antigênicos.

Tais informações mostram a importância do desenvolvimento de estudos que permitam uma rápida caracterização das cepas de $\mathbf{H}$. aegyptius quanto a sua capacidade invasora, em epidemias de conjuntivites purulentas por H. aegyptius.

Metodologias laboratoriais de maior sofislicação, até agora utilizadas para caractérizar as cepas de $\mathbf{H}$. aegyptius invasivas são de difícil aplicação em rotina de diagnóstico pelo alto custo e tempo necessário para a sua realização. Há portanto, a necessidade de se dispor de uma técnica rápida, de custo mais baixo que apresentem uma boa sensibilidade e especificidade, que possa ser utilizada para "screening" nas investigações epidemiológicas de surtos de conjuntivites por H. aegyptius.

A soroaglutinação em lāmina pela sua fácil 
BRANDILEONE, M.C.C.; VIEIRA, V.S.D.; TONDELLA, M.L.C.; SACCHI, C.T.; LANDGRAF, I.M.; ZANELLA, R.C.; BIBB, W.F.; IRINO, K. \& GRUPO DE ESTUDO DA FEBRE PURPÚRICA BRASILEIRA - Febre purpúrica brasileira. Caracierização rápida das cepas invasoras de Haemophilus aegyptius. Rev. Inst. Med. trop. S. Paulo, 31(4):221-227, 1989

reprodutibilidade, alta sensibilidade e especificidade já vem sendo utilizada com êxito na triagem de cepas invasivas de $\mathbf{H}$. aegyptius nos surtos de conjuntivite ocorridos no Estado de São Paulo. O fato do soro policlonal S-254 não apresentar reações cruzadas com outras espécies do gênero Haemophilus presentes na conjuntiva e orofaringe como foi observado na análise das 200 cepas pertencentes ao grupo 6 , demonstra a indicação de seu uso como método de diagnóstico presuntivo.

\section{CONCLUSÕES}

1) As cepas invasivas de $\mathbf{H}$. aegyptius podem ser caracterizadas através de seus antígenos de superfície, pelo método de aglutinação em lâmina com anti-soro policlonal específico.

2) O método de aglutinação em lâmina utilizando um anti-soro específico produzido com uma cepa de $\mathbf{H}$. aegyptius isolada de um caso de FPB apresentou $97,7 \%$ de sensibilidade e $89,6 \%$ de especificidade.

3) A boa reprodutibilidade de baixo custo da técnica de soroaglutinação em lâmina permite sua utilização como método de diagnóstico presuntivo, para icientificar cepas invasivas de $\mathbf{H}$. aegyptius responsáveis pela FPB, possibilitando a implantação de medidas adequadas na vigilância epidemiológica e prevenção desse agravo.

\section{SUMMARY}

Brazilian purpuric fever. Fast characterization of invasive Haemophilus aegyptius.

Strains of $\mathbf{H}$. aegyptius isolated during outbreak of Brazilian Purpuric Fever (BPF) in Brazil were characterized antigenically by slide agglutinaiion test utilizing antiserum produced with a $\mathbf{H}$. aegyptius strain isolated from blood culture from a patient with BPF. By means of this method, it were identified H. aegyptius strains responsible for outbreaks of conjunctivitis with identical antigenic characteristics to strains isolated from BPF. The sensitivity and specificity of slide seroagglutination test was $97.7 \%$ and $89.6 \%$ respectively; therefore this assay was efficient to be used as a screening method in the studies of purulent conjunctivitis for detecting high risk populations for BPF, and to implement measures that will increase the efficiency of epidemiologic surveillance.

\section{AGRADECIMENTOS}

Agradecemos o Dr. Lee Harrison, do Meningites and Special Pathogens, Division of Bacterial Diseases, Center for Disease Control, Atlanta, Estados Unidos, pelo envio das cepas do grupo 5.

\section{REFERÊNCIAS BIBLIOGRÁFICAS}

1. ALBRITTON, W.L. - Infections due to Haemophilus other than H. influenzae. Ann. Rev. Microbiol., 36:199-216, 1982.

2. ALBRITTON, W.L.; SETLOW, J.K.; THOMAS, M.; SOTTNEK, F. \& STEIGERWALT, A.G. - Heterospecific transformation in the genus Haemophilus. Molec. gen. Genet., 193:358-363, 1984.

3. BRAZILIAN PURPURIC FEVER TASK FORCE Preliminary report: epidemic fatal purpuric fever among children - Brazil. M.M.W.R., 34:217-219, 1985.

4. BRAZILIAN PURPURIC FEVER TASK FORCE Brazilian purpuric fever: Haemophilus aegyptius bacteremia complicating purulent conjunctivitis. M.M.W.R., 35:553-554, 1986.

5. BRAZILIAN PURPURIC FEVER STUDY GROUP - Brazilian purpuric iever: epidemic purpura fulminans associated with antecedent purulent conjunctivitis. Lancet 2:757-761, 1987.

6. BRAZILIAN PURPURIC FEVER STUDY GROUP - Haemophilus aegyptius bacteraemia in Brazilian purpuric fever. Lancet, 2:761-763, 1987.

7. BRENNER, D.J.; MAYER, L.W.; CARLONE, G.M.; HARRISON, L.H.; BIBB, W.F.; BRANDILEONE, M.C.C.; SOTTNEK, F.O.; IRINO, K.; REE- VES, M.W.; SWENSON, J.M.; BIRKNESS, K.A.; WEYANT, R.S.; BERKELY, S.F.; WOODS, T.C.; STEIGERWALT, A.G.; GRIMONT, P.A.D.; MCKINNEY, R.M.; FLEMING, D.W.; GHEESLING, L.L.; COOKSEY, R.C.; ARKO, R.Y.; BROOME, C.V. \& THE BRAZILIAN PURPURIC FEVER STUDY GROUP - Biochemical, genetic and epidemiologic characterization of Haemophilus influenzae biogroup aegyptius (Haemophilus aegyptius) strains associated with Brazilian purpuric fever. J. clin. Microbiol., 26:1524-1534, 1988.

8. DAVIS, D.J.; PITTMAN, M. \& GRIFFITS, J.J. Hemagglutination by the Koch-Weeks bacillus. J. Bact., 59:427-431, 1950.

9. FLEMING, D.W.; BERKELY, S.F.; BROOME, C.V.; BRANDILEONE, M.C.C.; WALDMAN, E.A. \& THE BRAZILIAN PURPURIC FEVER STUDY GROUP - Haemophilus aegyptius conjunctivitis associated with Brazilian purpuric fever. In: ANNUAL 
BRANDILEONE, M.C.C.; VIEIRA, V.S.D.; TONDELLA, M.L.C.; SACCHI, C.T.; LANDGRAF, I.M.; ZANELLA, R.C.; BIBB, W.F.; IRINO, K. \& GRUPO DE ESTUDO DA FEBRE PURPÜRICA BRASILEIRA - Febre purpúrica brasilcira. Caracterização rápida das cepas invasoras de Haemophilus aegyptius. Rev. Inst. Med. trop. S. Paulo, 31(4):221-227, 1989.

MEETING OF THE AMERICAN SOCIETY FOR MICROBIOlOGY, 88. Washington, DC, 1986. Program and abstracts. Abstr. $\mathrm{C} 2$.

10. HARRELL, W.K.; ASHWORTH, H.; BRITT, L.E.; GEORGE, J.R.; GRAY Jr., S.B.; GREEN, J.H.; GROSS, H. \& JOHNSON, J.E. - Procedural manual of production of bacterial, fungal and parasitic reagents. Atlanta, Biological Reagents Section, Laboratory Division, Centers for Disease Control, 1970. p.1820.

11. IRINO, K.; LEE, I.M.L.; KAKU, M.; BRANDILEONE, M.C.C.; MELLES, C.E.A.; LEVY, C.E.; BERKLEY, S.E.; FLEMING, D.W.; SILVA, G.A. \& HARRISON, L. - Febre purpúrica brasileira: resultados preliminares da investigação etiológica. Rev. Inst. Med, trop. S. Paulo, 29:174-177, 1987.

12. IRINO, K.; GRIMONT, F.; CASIN, I.; GRIMONT, P.A.D. \& THE BRAZILIAN PURPURIC FEVER STUDY GROUP - rDNA gene restriction patterns of Haemophilus influenzae biogroup aegyptius strains associated with the Brazilian purpuric fever. J. clin. Microbiol., 26:1535-1538, 1988.

13. KILIAN, M. \& BIBERSTEIN, E.L. - Haemophilus Winslow, Broadhurst, Buchanan, Krumwiede, Robers and Smith, 1917. In: KRIEG, N.R.\& HOLT, J.G., ed. - Bergey's manual of systematic bacteriology. Baltimore, Williams \& Wilkins, 1984, v.1, p.558-569.

14. LEIDY, G.; HAHN, E. \& ALEXANDER, H.E. - Interspecific transformation in Haemophilus: a possible index of relationship between $\boldsymbol{H}$. influenzae and $\mathbf{H}$. aegyptius. Proc. Soc. exp. Biol. (N.Y.), 102:86-88, 1959.

15. LEIDY, G.; JAFFEE, I. \& ALEXANDER, H.E. Further evidence of a high degree of genetic homology between $\mathbf{H}$. influenzae and $\mathbf{H}$. aegyptius. Proc. Soc. exp. Biol. (N.Y.), 118:671-679, 1964.

16. MAZLOUM, H.A.; KILIAN, M.; MOHAMED, Z. \& SAID, M.D. - Differentiation of Haemophilus aegyptius and Haemophylus influenzae. Acta path. microbiol. scand., 90:109-112, 1982.

17. MCINTYRE, P.; WHEATON, G.; ERLICH, J. \& HANSMAN, D. - Brazilian purpuric fever in central Australia. Lancet, 2:112, 1987.

18. PITTMAN, M. - Variation and type specificity in bacterial species Haemophilus influenzae. J. exp. Med., 53:471-492, 1931.

19. PITTMAN, M. \& DAVIS, D.J. - Identification of the Koch-Weeks Bacillus (Haemophilus aegyptius). J. Bact., 59:413-426, 1950.

20. POHL, S. - DNA relatedness among members of Actinobacillus: Haemophilus and Pasteurellae. In: KILLIAN, M.; FREDERIKSEN, W. \& BIBERSTEIN, E.L., ed. - Haemophilus, Pasteurella and Actinobacillus. London, Academic Press, 1981. p.245-253.

21. PORATH, A.; WANDERMAN, K.; SIMU, A.; VIDNE, B. \& ALKAN, M. - Case report: endocarditis caused by Haemophilus aegyptius. Amer. J. med. Sci., 292:110-111, 1986 .

Recebido para publicação em 20/12/1988. 